\title{
Comparison of Serum Matrix Metalloproteinase-9 Value in Spondylitis Tuberculous with Degenerative Spine Disease
}

\author{
Otman Siregar $^{1 *}$, Yohanes Augustinus ${ }^{2}$, Benny Benny ${ }^{1}$, Ahmad Jabir Rahyussalim $^{3}$ \\ ${ }^{1}$ Spine Division, Faculty of Medicine, Universitas Sumatera Utara, Haji Adam Malik Hospital, Medan, Indonesia; ${ }^{2}$ Faculty of \\ Medicine Universitas Sumatera Utara/Haji Adam Malik Hospital-Medan; ${ }^{3}$ Faculty of Medicine, Universitas Indonesia, Cipto \\ Mangunkusumo Hospital, Jakarta, Indonesia
}

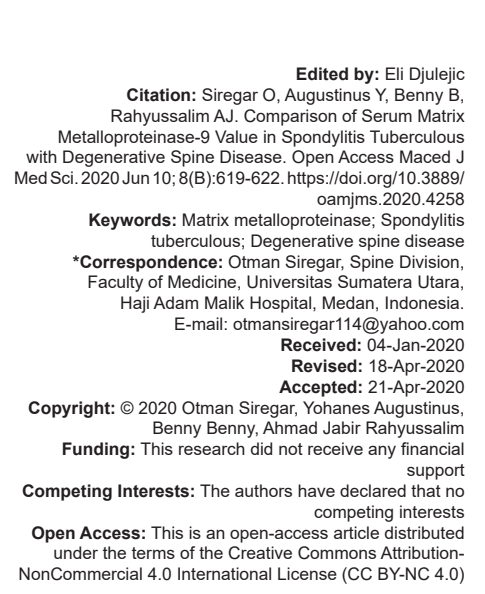

\section{Abstract}

AIM: We aimed to evaluate and compare the expression of MMP-9 in TB spondylitis using serum levels in the blood of patients suffering from TB spondylitis and compared to the control group; in this case, the control group in this study was patients with degenerative diseases of the spine.

METHODS: Fourteen subjects were divided into two groups, with seven subjects of spondylitis tuberculous (TB) (ST) and seven subjects of degenerative spine disease (DSD) in the period from December 2017 to November 2018 who were included in the inclusion criteria included in this study and blood sampling was taken for the examination of serum matrix metalloproteinase-9 (MMP-9) levels.

RESULTS: There were significant differences in serum MMP-9 levels between ST and DSDs with a significance value of $0.002(p<0.05)$ with low serum MMP-9 levels in the ST study group $1857.14 \pm 377.96$ and mean in the control group $857.14 \pm 243.97$. There were significant differences in serum MMP-9 levels between ST and DSDs with a significance value of $0.002(p<0.05)$ with low serum MMP-9 levels in the ST study group $1857.14 \pm 377.96$ and mean in the control group $857.14 \pm 243.97$

CONCLUSION: Patients suffering from ST have higher serum MMP-9 levels than patients with DSD, although MMP-9 is not a specific marker examination for ST, the results of this study can be suggestive into that can help to evaluate enzyme activity in patients with ST disease.

\section{Introduction}

Tuberculous (TB) is one of the long-known diseases and is still the leading cause of death in the world. The prevalence of TB in Indonesia and other developing countries is quite high. In 2006, new cases in Indonesia amounted to more than 600,000 and most of them were suffered by people in productive age (15-55 years).

About $20 \%$ of infections with pulmonary TB will spread out of the lungs (extrapulmonary TB). Eleven percent of extrapulmonary TB is osteoarticular TB, and nearly half of patients suffer from spinal TB infection. Half have lesions in the spine with neurological deficits of $10-45 \%$ of sufferers.

Spondylitis TB (ST) is an infection of the spine caused by Mycobacterium tuberculosis [1], [2], [3] ST results in damage to the body in the form of a defect that causes spinal instability and disruption of surrounding structures [1], [3] The occurrence of infection in ST can originate from primary infection [2], [4] bacteria directly infect the corpus, or secondary infections [4], namely, bacteria spread hematogenously or lymphogenously from the location of the primary disease to the spinal cord [2]. Infections that occur in ST are generally secondary infections of the lungs, but in some cases are primary infections. Pro-inflammatory and antiinflammatory cytokines play an important role in the development and control of M. tuberculosis infection [5]. It has been demonstrated that cytokine profiles will differ in each degree of disease [1], [5]. Several cytokines have been known to be biomarkers of disease activity in TB infections. Matrix metalloproteinase (MMP) is a zinc-dependent protease, which plays a role in the process of degradation of the extracellular matrix and modulates the inflammatory response by facilitating and inhibiting different cytokines [6], [7].

In determining the form of treatment for ST, it is generally divided into two groups of patients, namely, groups of patients accompanied by complications in the way of neurological disorders and groups that are not accompanied by neurological disorders. In patients without neurological complications, medical treatment is the primary choice in treatment, and surgical treatment is only needed in some special cases. However, in patients are accompanied by neurological complications, the combination of medical treatment and surgical management is the most appropriate treatment. 
MMP is a zinc-dependent proteinase that has an important role in the degradation and rotation of the extracellular matrix [3]. Since it was first reported, 24 members of MMP have been identified with specificity and function of overlapping substrates. Following each substrate and function specification, MMP is divided into six classes: Stromelysins, collagenases, matrilysins, gelatinases, membrane-type MMPs, and others.

Two gelatinases, MMP-2 and MMP-9 can reduce original type IV collagen and denaturation of type I collagen (gelatin). Both circulation and resident inflammatory cells can synthesize MMP-9. Experimental studies have provided evidence that MMP-9 levels were significantly higher in bronchoalveolar fluid patients with cavitary active TB, and lung extracts of mice infected with M. tuberculosis, compared to the control group. An increased significance of MMP-9 was also observed in TB cerebrospinal fluid (CSF) meningitis patients and also compared with people suffering from viral meningitis, where usually these enzymes are generally not found in CSF.

Many previous studies have shown that M. tuberculosis can stimulate MMP-9 expression in the lungs of infected organisms, but no studies have examined how the expression of MMP-9 in TB spondylitis when compared to the control group. Therefore, researchers are interested in trying to evaluate and compare the expression of MMP-9 in TB spondylitis using serum levels in the blood of patients suffering from TB spondylitis and compared to the control group; in this case, the control group in this study was patients with degenerative diseases of the spine.

\section{Methods}

This cross-sectional study was conducted at the Faculty of Medicine, Universitas Sumatra Utara/ Haji Adam Malik Hospital, North Sumatra, Indonesia, for 11 months from December 2017 to November 2018 by taking patient data and examining serum values of MMP-9, 14 subjects met the inclusion criteria.

Patients met the inclusion criteria recorded age, sex, and blood tests to determine serum MMP-9 levels.

The study sample was divided consecutively with equal numbers into two groups, namely: ST, and degenerative spine disease (DSD). Patients were obtained from outpatient or inpatient care diagnosed with ST and DSD. Patients who were included in the inclusion criteria were taken for blood sampling to examine levels of MMP-9.

The MMP-9 examination in this study used MMP-9 ELISA Kit from fine test with the catalog number ERB0080, size $48 \mathrm{~T} / 96 \mathrm{~T}$ and reactivity to humans. The scale value used is $3.125-200 \mathrm{ng} / \mathrm{ml}$ with sensitivity: $<1.875 \mathrm{ng} / \mathrm{ml}$. The application of the dosage is a quantitative detection of MMP-9 in serum, plasma, tissue homogenates, and other biological fluids. The differences in serum MMP-9 values in ST with the degenerative disease in the spine were analyzed using the Mann-Whitney test because the data obtained were abnormally distributed. All statistical calculations are carried out using a computer-based statistical program. The study was approved by the Health Research Ethics Committee of the Medical Faculty of the University of North Sumatra/Haji Adam Malik Hospital, and informed consent was obtained from all subjects.

\section{Results}

Before discussing the results of the study, because this study had no references, it was conducted with a small-scale preliminary study using ten balanced numbers of research subjects (five subjects with ST, and five subjects with DSD) with women as many as six people (60\%) and men as many as four people (40\%), with a mean and standard deviation of $41.6 \pm 18.8$ years, while the mean of subjects with ST $1800 \pm 447.2$ and mean DSD $800 \pm 273.8$ (Table 1).

Table 1: Preliminary study distribution

\begin{tabular}{|c|c|c|}
\hline \multirow[t]{2}{*}{ Variable } & \multicolumn{2}{|l|}{ Disease } \\
\hline & ST & DSD \\
\hline Gender M/F & $1 / 4$ & $3 / 2$ \\
\hline MMP-9 $(\mathrm{nm} / \mathrm{ml})$ & $1800 \pm 447.2$ & $800 \pm 273.8$ \\
\hline Mean age & $41.6 \pm 18.8$ & \\
\hline
\end{tabular}
the final analysis. Data retrieval and examination of serum MMP-9 values in ST and DSD were carried out in stages with the initial phase of selecting samples of subjects included in the inclusion criteria. Seven subjects with diagnosed ST, seven subjects with diagnoses of DSD, eight women $(57.1 \%)$, and six men $(42.9 \%), 13$ years old are the youngest research subjects and 73 years old oldest subjects with mean and standard deviations amounting to $44.79 \pm$ 16.98 years. From the results of the analysis, it was found that the mean and standard deviation of serum ST MMP-9 values was $1857.14 \pm 377.96$ while the mean and standard deviation of serum DSD levels of MMP-9 was $857.14 \pm 243.97$ (Table 2).

Table 2: Study subject distribution

\begin{tabular}{lll}
\hline Variable & Disease & \\
\cline { 2 - 3 } & ST MMP-9 $(\mathrm{nm} / \mathrm{ml})$ & DSD MMP-9 $(\mathrm{nm} / \mathrm{ml})$ \\
\hline Patient & 2000 & 1000 \\
1 & 1000 & 1000 \\
2 & 2000 & 500 \\
3 & 2000 & 500 \\
4 & 2000 & 1000 \\
5 & 2000 & 1000 \\
6 & 2000 & 1000 \\
7 & $1857.14 \pm 377.96$ & $857.14 \pm 243.97$ \\
Mean MMP-9 $(\mathrm{nm} / \mathrm{ml}) \pm$ SD & 0.002 & 43 year old \\
p-value & 13 year old & 73 year old \\
Youngest age & 62 year old & 0 \\
Oldest age & $9.14 \pm 5.29$ weeks \\
TB drug consumption & & \\
\hline ST: Spondylitis tuberculous, DSD: Degenerative spine disease, n: Subject. MMP-9: Matrix \\
metalloproteinase-9, TB: Tuberculous.
\end{tabular}


From the results of the statistical analysis of the comparison of serum MMP-9 values in ST with DSD, the results were significant that the serum MMP-9 values were higher and this was indicated by $p=0.002$ $(p<0.05)$.

\section{Discussion}

Pott's disease is the most common spinal granulomatous bacterial infection and is the most frequent bone TB [5]. The occurrence of infection in ST can originate from primary infection [2], [4], i.e. bacteria directly infect the corpus, or secondary infections [6], namely, bacteria spread hematogenously or lymphogenic from the location of the primary infection to the spinal cord [2]. Infections that occur in ST are generally secondary infections of the lungs, but in some cases are primary infections. Pro-inflammatory and anti-inflammatory cytokines play an important role in the development and control of $M$. tuberculosis infection [5]. It has been demonstrated that cytokine profiles will differ in each degree of [1], [4] Several cytokines have been known to be biomarkers of disease activity in TB infections. MMP is a zinc-dependent protease, which plays a role in the process of degradation of the extracellular matrix and modulates the inflammatory response by facilitating and inhibiting different cytokines [6].

Two gelatinases, MMP-2 and MMP-9 have the ability to reduce original type IV collagen and denaturation of type I collagen (gelatin). Both circulation and resident inflammatory cells have the capacity to synthesize MMP-9. Experimental studies have provided evidence that MMP-9 levels were significantly higher in bronchoalveolar fluid patients with cavitary active TB, and lung extracts of mice infected with M. tuberculosis, compared to the control group.

In a study conducted by Hrabec et al., 2002 [8], it was found that serum MMP-9 levels in patients with active pulmonary TB had significantly higher levels of the control group with mean MMP-9 levels in the group with pulmonary TB $1.23 \pm 0.43 \mu \mathrm{U} / \mu \mathrm{L}$, while the mean MMP-9 level in the control group was $0.37 \pm 0.10 \mu \mathrm{U} / \mu \mathrm{L}$ and this result was in accordance with the function of MMP-9 which could induce enzymes produced by mononuclear phagocytes and stimulated neutrophils. This finding is in accordance with the study conducted that there was a significant difference in MMP-9 levels in patients with ST with a control group used in this group with subjects with DSD with mean ST patients $1857.14 \pm 377.96$ while the mean in patients with DSD $857.14 \pm 243.97$ with $p=0.002(p<0.05)$, but in this study, there was no comparison of the ratio of white blood cell levels in the two study groups as did the research conducted by Hrabec et al., 2002, with this study obtained MMP-9 rate with an increase in white blood cells was significantly higher in the study group with pulmonary TB $0.150 \pm 0.054 \mathrm{mU} / \mathrm{ml} / \mathrm{L}$ while in the control group $0.059 \pm 0.023 \mathrm{mU} / \mathrm{ml} / \mathrm{L}$. In this study, no correlation was found between the duration of consumption of anti-TB drug and an increase in serum MMP-9 levels $(p>0.05)$ with an average use for TB medicines $9.14 \pm 5.29$ weeks, although this was not a primary goal in this study, need to be reviewed, because this assessment only uses seven research subjects [9], [10], [11].

\section{Conclusion}

From the results of the comparison of serum MMP-9 values in ST with DSD, it was found that the serum MMP-9 values in ST gave higher results so that MMP-9 examination could be used as a barometer for the diagnosis of ST.

Further studies are needed to find out whether the increase in serum MMP-9 levels in TB spondylitis is also accompanied by an increase in the ratio of white blood cells and further studies are needed to determine whether an increase in serum MMP-9 levels can also be influenced by the duration of use of anti-TB drugs.

\section{References}

1. Lindsay, KW, Bone I, dan Calldaner R. Spinal cord and roo compression. In: Neurology and Neurosurgery Illustrated. $2^{\text {nd }}$ ed. Edinburgh: Churchill Livingstone; 1991. p. 388.

2. Martini $\mathrm{FH}$, Welch $\mathrm{K}$. The lymphatic system and Immunity. In: Fundamentals of Anatomy and Physiology. $5^{\text {th }}$ ed. New Jersey: Upper Saddle River; 2001. p. 132-51.

3. Tachdjian MO. Tuberculosis of the spine. In: Pediatric Orthopedics. $2^{\text {nd }}$ ed. Philadelphia, PA: W.B. Saunders; 1990. p. 1449-54

4 Graham JM, Kozak J. Spinal tuberculosis. In: Hochschule SH, Cotler HB, Guyer RD, editors. Rehabilitation of the Spine: Science and Practice. St. Louis: Mosby-Year Book, Inc.; 1993. p. 387-90.

5. Patil T, Garg RK, Jain A, Goel MM, Malhotra HS, Verma R, et al. Serum and CSF cytokines and matrix metalloproteinases in spinal tuberculosis. Inflamm Res. 2015;64(2):97-106. https:// doi.org/10.1007/s00011-014-0786-5.

PMid:25503789

6. Salgame P. MMPs in tuberculosis: Granuloma creators and tissue destroyers. J Clin Invest. 2011;121(5):1686-8. https://doi. org/10.1172/jci57423

PMid:21519148

7. Agarwal P, Rathi P, Verma R, Pradhan CG. Tuberculous Spondylitis: "Global lesion". Special Issues on Tuberkulosa. Bombay: Bombay Hospital Journal; 1999.

8. Hrabec E, Strek M, Zieba M, Kwiatkowska S, Hrabec Z. Circulation level of matrix metalloproteinase- 9 is correlated with 
disease severity in tuberculosis patients. Int J Tuberc Lung Dis. 2002;6(8):713-9.

PMid:12150484

9. Leibert E, Haralambou G. Tuberkulosa. In: Rom WN, Garay S editors. Spinal Tuberculosis. Philadelphia, PA: Lippincott, Williams, and Wilkins; 2004.

10. López-Otín C, Palavalli LH, Samuels Y. Protective roles of matrix metalloproteinases: From mouse models to human cancer. Cell Cycle. 2009;8(22):3657-62. https://doi.org/10.4161/ cc.8.22.9956

PMid: 19844170

11. Singh S, Dawar H, Das K, Mohapatra B, Prasad S. Functional and radiological outcomes of anterior decompression and posterior stabilization via posterior transpedicular approach in thoracic and thoracolumbar Pott's disease: A retrospective study. Asian Spine J. 2017;11(4):618-26. https://doi.org/10.4184/ asj.2017.11.4.618

PMid:28874981 